\title{
Assessment of selected psychological factors in patients with inflammatory bowel disease
}

\author{
Joanna M. Morys ${ }^{1,2}$, Anna Kaczówka, Maria Jeżewska ${ }^{4}$ \\ ${ }^{1}$ Department of Clinical Psychology, Medical University of Gdansk, Gdansk, Poland \\ ${ }^{2} 1^{\text {st }}$ Cardiology Clinic, Medical University of Gdansk, Gdansk, Poland \\ ${ }^{3}$ Regional Specialist Hospital, Olsztyn, Poland \\ ${ }^{4}$ Department of Occupational Diseases and Internal Affairs, Medical University of Gdansk, Gdansk, Poland
}

Prz Gastroenterol 2016; 11 (1): 47-53

DOI: $10.5114 /$ pg.2015.52560

Key words: psychological factors, ulcerative colitis, Crohn's disease.

Address for correspondence: Joanna M. Morys PhD, Department of Clinical Psychology, Medical University of Gdansk, 15 Tuwima St, 80-210 Gdansk, Poland, phone: +48 501081 544, e-mail: jmb@gumed.edu.pl

\begin{abstract}
Introduction: In the majority of patients with inflammatory bowel diseases (IBD) mental health problems are demonstrated in the form of excessive anxiety and unstable emotional reactions. Emotional instability affects the wellbeing of these patients, and also their attitude towards the disease and the course of treatment.

Aim: The objective of the study is to evaluate psychological factors in patients with IBD, and to compare patients with ulcerative colitis (UC) and patients suffering from Crohn's disease (CD) with respect to intensity of these factors.

Material and methods: The study included 108 patients with IBD. NEO-FFI and type-D Scale were used to evaluate personality traits. Anxiety level was measured with STAI. CECS was used to measure the patients' tendency to suppress negative emotions.

Results: In the patients with CD $45 \%$ of subjects presented type D personality. In the group of patients with UC, type D personality was found in $38 \%$. The higher levels of neuroticism and extraversion were observed in patients with UC, whereas the patients with CD exhibited a higher level of openness to experience and agreeableness. In patients with CD the levels of state anxiety were elevated, whereas in patients with UC higher scores were both for state and trait anxiety.

Conclusions: There were no statistically significant differences in terms of personality factors in a group of patients with CD and UC. It was found that the majority of patients with IBD suffer elevated or high sense of anxiety.
\end{abstract}

\section{Introduction}

Inflammatory bowel diseases are classified as chronic diseases in which psychological factors play a significant role. The results of previous studies on patients with inflammatory bowel disease (IBD) showed that in the majority of patients mental health problems are demonstrated in the form of excessive anxiety and unstable emotional reactions [1]. Emotional instability affects the wellbeing of these patients, and also their attitude towards the disease and the course of treatment $[2,3]$. The aetiology of inflammatory bowel diseases has been insufficiently explained [4]. However, environmental factors, genetic predisposition, and some immune aspects have been found to cause the disease.

The environmental factors influencing the occurrence of IBD are commonly reported as socio-economic development [4]. The incidence and prevalence of both Crohn's disease (CD) and ulcerative colitis (UC) are high, especially in developed countries as these diseases are associated with bad eating habits, accessibility of vaccines and antibiotics, elimination of helminth ova, and decreased number of infectious agents in everyday life. All these phenomena mean a reduced stimulation of the immune system at the early stages of human development, which in turn increase the probability of the occurrence of immune diseases at subsequent stages of life.

Mental stress accompanied by haste is present every day, mainly in Western civilisations, and it affects both the pathogenesis of IBD and the pace of disease progression $[5,6]$. Some studies suggested that chronic stress correlates with subclinical elevation of any inflammation in the intestine. Analyses carried out on middle-aged or elderly subjects who had been under stress revealed el- 
evated levels of C-reactive protein in their blood. Also, there were reports in which the concentration of proinflammatory cytokines - essential in IBD aetiology was increased, namely: tumor necrosis factor $\alpha$ (TNF- $\alpha)$, interleukin 6, 2 (IL-6, IL-2), and interferon $\gamma$ (INF- $\gamma$ ) [7].

Smoking is a major risk factor among patients with $C D$, and it is significant in the course of IBD. Smoking affects both an activity and phenotypic manifestation of the disease. In smokers with CD, the inflammatory processes often affect the ileum but somehow leave the large intestine intact. Smoking does not increase symptoms in UC patients. Some reports indicate that in UC patients smoking may reduce the risk of symptomatology appearance by as much as $40 \%$ [8].

Studies on the psychological aspects of IBD conducted in the 1950 s and 1960 s indicate that $60 \%$ of new cases or relapses of UC were preceded by "depressive life situations" and/or "the onset and later exacerbation of this disease had followed a catastrophic experience" [9]. It was believed that "these patients came from the family of symbiotic structure mainly", and that they were characterised by low self-esteem and a tendency to profoundly experience any failure.

Until now, there has been no clarity regarding the impact of psychological factors on IBD. Many studies have included both CD and UC subjects in the same clinical group of inflammatory bowel diseases. This approach might have brought inaccuracies in the results. Some authors who investigated personality dimensions indicated that personality might have led to physiological changes [10]. According to them the diseases mentioned above might have been brought by factors such as depression, anxiety, A behaviour pattern, hostility syndrome, and D type personality [11].

In some studies, patients with IBD (both CD and UC) revealed elevated levels of CRP and pro-inflammatory cytokines, if they demonstrated high levels of neuroticism and low acceptance of disease [12]. High levels of neuroticism resulted in poorer adaptation to life, and they more strongly intensified negative emotional reactions towards IBD symptoms. Analysis of the personality traits in each stage of the disease (exacerbation/ remission) showed insignificant differences in the level of personality traits, namely in neuroticism, openness to experience, conscientiousness, agreeableness, and extraversion at the stage of exacerbation. Another report suggested that patients who presented with CD manifested more neurotic or depressive symptoms, suffered stronger fears, and had lower vitality than UC patients [13].

\section{Aim}

The primary objective of this study is to evaluate selected psychological factors in patients with IBD. The secondary objective is to compare patients with UC and patients suffering from $C D$, with respect to the intensity of these factors.

\section{Material and methods \\ Subjects}

The study included 108 consecutive patients with inflammatory bowel disease - CD or UC. The study was conducted from June 2011 to February 2012 in the Gastroenterology Department of the Regional Hospital in Olsztyn, and was supplemented by on-line questionnaires collected via the official net - a forum of patients with IBD. All subjects consented to participate in the study of their own accord. After becoming familiar with the procedure of testing the participants received a printout with questionnaires (hospital in-patients) or an electronic one (net-forum participants). Participants filled in the questionnaires unassisted. The subjects were provided with the necessary instructions for the completion of questionnaires. If a participant misunderstood any instruction, a researcher provided an explanation.

In patients with UC $24 \%$ were diagnosed within a year before the examination, another $24 \%$ were diagnosed from year to 3 years before the examination, $14 \%$ had suffered from UC from 3 to 5 years, $22 \%$ from 5 to 10 years, and $16 \%$ of patients had suffered for more than 10 years. In patients with CD 9\% were diagnosed within a year prior to the study, $14 \%$ were diagnosed from year to 3 years before the examination, $21 \%$ had suffered from UC from 3 to 5 years, 34\% from 5 to 10 years, and $22 \%$ of patients for more than 10 years.

Patients with CD were more frequently hospitalised due to IBD than those with UC. In patients with CD 9\% were hospitalised more than 20 times, $13 \%$ were hospitalised from 11 to 20 times, 21 patients were hospitalised $6-10$ times, 53\% 1-5 times, and 5\% of patients were never hospitalised. In patients with UC the largest group of patients were hospitalised from 1 to 5 times $-82 \%, 6 \%$ were hospitalised from 11 to 20 times, and $12 \%$ had no previous hospitalisations.

Patients were treated with standard drugs: corticosteroids, amino salicylate medicine (Sulfasalazine, Mesalazine), immunosuppressant: Azathioprine, Mercaptopurine, 'biological'/'anti-TNF' drugs Remicade (infliximab) and Humira (adalimumab). Most patients had no comorbidities. Only $20 \%$ of the CD group and $22 \%$ in UC reported comorbidities: osteoporosis, arthritis (4\% - CD and 5\% - UC), autoimmune diseases (celiac disease, systemic lupus erythematosus, psoriasis, multiple sclerosis; $5 \%$ in CD and 4\% in UC) and other diseases: hypertension, diabetes, thyroid or ovarian disease, and depressive disorders (11\% in CD and 13\% in UC). 


\section{Methods}

A demographic questionnaire was used in the study. It consisted of a set of 10 questions about: age, gender, education, place of residence, civil status, duration of illness, number of hospital stays, relationship, traumatic events, onset of the disease, and comorbid diseases. The last question concerned the quality of life. Each patient was asked to mark their answers on a 10-point scale, from 1 (lowest possible quality) to 10 (highest possible quality).

Five Factor Inventory (NEO-FFI) and Type-D Scale were used to evaluate personality traits. Anxiety levels were measured with the State-Train Anxiety Inventory. The Courtauld Emotional Control Scale was used to measure the patients' tendency to suppress negative emotions.

The NEO Five Factor Inventory (NEO-FFI) was developed by Costa and McCrae. It identifies five personality traits, called the "Big Five". According to this model the structure of personality consists of five dimensions described as neuroticism, openness to experience, extraversion, agreeableness, and conscientiousness. It is assumed that the personality traits defined in the "Big Five" are largely innate and reflect human biology rather than life experiences.

The Type-D Scale (DS-14) is to test "distressed personality" known as "Type D personality". Distressed personality involves two dimensions: social inhibition and negative affectivity. These dimensions are regarded as relatively stable personality traits. Negative affectivity is expressed in dysphoria, worrying, and irritability. Social inhibition refers to refraining from expression of negative emotions and behaviours associated with these emotions. The inhibition of expression of negative emotions is mainly manifested in social situations. It is based on the fear of being rejected by others and the unawareness of an individual.

The State and Trait Anxiety Inventory was developed by Spielberger as a tool for investigating states of anxiety (SSAI), and traits of anxiety as a personal characteristic (STAI).

The Courtauld Emotional Control Scale (CECS), developed by M. Watson and S. Greer, was applied to measure personal ability to control major emotions, such as anger, depression, and anxiety.

\section{Statistical analysis}

Hypotheses verification was performed with the use of ANOVA analysis. All data were collected in a spreadsheet program Microsoft Office Excel 2003. Data analysis was performed using the statistical package, Statistica 10.

\section{Results}

The scores from the assessment of quality of life expressed in a 10-point scale were higher in patients with CD $(80 \%$ of patients identified the quality of life of $\geq 6$ points) than in patients with UC ( $68 \%$ pointed to $\geq 6$ ) (Table I).

In the group of patients with CD $45 \%$ of subjects presented type $D$ personality, while $55 \%$ did not have $D$ type personality (Table II). In the group of patients with UC, type D personality was found in $38 \%$ of patients while $62 \%$ did not exhibit type $D$ personality traits. A large amount of SI (>10) was observed in $79 \%$ of patients with CD and in $80 \%$ of patients with UC. In the second dimension of the type $D$ personality - negative emotionality, a high level of features was observed in $62 \%$ of patients with CD and in $70 \%$ with UC.

The assessment of the "Big Five" traits indicates that higher levels of neuroticism and extraversion were observed in patients with UC, whereas the patients with $C D$ exhibited a higher level of openness to experience and agreeableness (Table III). Although these differences were found to exist, they were not statistically significant. Results referring to the conscientiousness scale were similar in both groups.

In patients with $C D$ the levels of state anxiety were elevated, whereas in patients with UC higher scores were for State and Trait anxiety. Yet again these differences were not statistically significant (Table IV).

The assessment of emotion control shows similar results in both groups of anger and depression control (Table V). However, in the case of control of anxiety higher results were obtained by patients with CD. But these were not statistically significant differences.

\section{Discussion}

An increased interest in the relationship between personality and somatic diseases occurred in the late 1990s, and it has been growing ever since. Current research on the influence of personality on the development of diseases is a frequent subject of scientific discussion. Nowadays, in addition to a purely clinical approach, the psychological and social contexts of the diseases are considered. There is a fairly substantial amount of evidence on the relationship between the personality and health of an individual. Some personality traits may contribute to health, while others may prompt the development of disease [14].

An important indicator of both physical and mental health is neuroticism, included in the "Big Five" model. Research has shown that neuroticism affects the amount of perceived physical symptoms and unpleasant symptoms associated with wellbeing and the quality of life. It has been proven, moreover, that the expe- 
Table I. Socio-demographic characteristics of the study group

\begin{tabular}{|c|c|c|c|c|c|}
\hline \multirow[t]{2}{*}{ Parameter } & & \multicolumn{2}{|c|}{$\mathrm{CD}(N=58)$} & \multicolumn{2}{|c|}{$\mathrm{UC}(N=50)$} \\
\hline & & $n$ & $\%$ & $n$ & $\%$ \\
\hline \multirow[t]{4}{*}{ Age [years] } & $17-30$ & 40 & 69 & 26 & 52 \\
\hline & $31-40$ & 11 & 19 & 11 & 22 \\
\hline & $41-50$ & 5 & 9 & 5 & 10 \\
\hline & $51-64$ & 2 & 3 & 8 & 16 \\
\hline \multirow[t]{2}{*}{ Gender } & Female & 37 & 64 & 31 & 62 \\
\hline & Male & 21 & 36 & 19 & 38 \\
\hline \multirow[t]{4}{*}{ Education } & Elementary & 3 & 5 & 1 & 2 \\
\hline & Secondary & 27 & 47 & 22 & 44 \\
\hline & Incomplete higher & 11 & 19 & 8 & 16 \\
\hline & Higher & 17 & 29 & 19 & 38 \\
\hline \multirow[t]{5}{*}{ Domicile } & Village & 13 & 22 & 15 & 30 \\
\hline & Small town up to 10 thousand & 3 & 5 & 4 & 8 \\
\hline & Town from 10 thousand to 100 thousand & 16 & 28 & 11 & 22 \\
\hline & City from 100 thousand to 500 thousand & 12 & 21 & 16 & 32 \\
\hline & City of over 500 thousand & 14 & 24 & 4 & 8 \\
\hline \multirow[t]{4}{*}{ Marital status } & Single & 32 & 55 & 21 & 42 \\
\hline & Married & 18 & 31 & 22 & 44 \\
\hline & Widow/widower & 0 & 0 & 1 & 2 \\
\hline & Informal relationship & 8 & 14 & 6 & 12 \\
\hline
\end{tabular}

CD - Crohn's disease, UC-colitis ulcerosa.

Table II. Assessment of the type D personality and its two dimensions: social inhibition and negative affectivity in patients with CD and UC

\begin{tabular}{lcccccc} 
Disease & \multicolumn{2}{c}{ Type D } & \multicolumn{2}{c}{ SI } & \multicolumn{2}{c}{ NA } \\
\cline { 2 - 7 } & $<10$ & $>10$ & $<10$ & $>10$ & $<10$ & $>10$ \\
\hline CD & 55 & 45 & 21 & 79 & 38 & 62 \\
\hline UC & 62 & 38 & 20 & 80 & 30 & 70
\end{tabular}

Type D - Type D personality, SI - social inhibition, NA - negative affectivity. rience of negative emotions which are characteristic of individuals with high levels of neuroticism may impair the health of patients [15]. In some illnesses neuroticism may be a predictor of diseases (coronary heart disease, ulcers, psoriasis, cancer) [16].

Our studies performed in patients with inflammatory bowel disease have shown that more than $35 \%$ of the respondents manifested high levels of neuroticism.

Despite the lack of statistically significant differences in the level of neuroticism in patients with $C D$

Table III. Intensity of the "Big Five": neuroticism, openness to experience, extraversion, agreeableness, and conscientiousness, in patients with CD and UC

\begin{tabular}{|c|c|c|c|c|c|c|c|c|c|c|c|c|c|c|c|}
\hline \multirow[t]{2}{*}{ Disease } & \multicolumn{3}{|c|}{ Neuroticism } & \multicolumn{3}{|c|}{ Openness to experience } & \multicolumn{3}{|c|}{ Extraversion } & \multicolumn{3}{|c|}{ Agreeableness } & \multicolumn{3}{|c|}{ Conscientiousness } \\
\hline & $\mathrm{L}$ & $M$ & $\mathrm{H}$ & $\mathrm{L}$ & $M$ & $\mathrm{H}$ & $\mathrm{L}$ & $M$ & $\mathrm{H}$ & $\mathrm{L}$ & $M$ & $\mathrm{H}$ & $\mathrm{L}$ & $M$ & $\mathrm{H}$ \\
\hline $\mathrm{CD}[\%]$ & 28 & 36 & 36 & 20 & 39 & 41 & 27 & 50 & 23 & 10 & 45 & 45 & 8 & 45 & 47 \\
\hline UC [\%] & 14 & 48 & 38 & 24 & 48 & 28 & 22 & 52 & 26 & 16 & 50 & 34 & 12 & 42 & 46 \\
\hline
\end{tabular}

L-Low level of trait (1-3 sten), $M$ - moderate level of trait (4-6 sten), $\mathrm{H}$ - high level of trait (7-10 sten). 
and UC, it was possible to notice a greater tendency for higher scores in the UC group. This may be explained by the fact that patients with UC reported a shorter duration of their illness, fewer hospital stays, and better opportunities associated with the disease. The author assessed their quality of life higher compared to the patients with CD, whose outcome is inherently much worse.

Neuroticism, in addition to negative attitudes to life, binds with a low resistance to stress and life events experienced. Moreover, difficult situations, as evidenced, can lead to a reduction of CD8, NK cells, and macrophages in the blood, lowering immunity. Chronic stress is also associated with subclinical inflammation in the intestine [17]. Other studies showed elevated levels of C-reactive protein (CRP) and pro-inflammatory cytokines in patients with IBD, who were characterised by a high level of neuroticism [13]. It can be concluded that the combination of neuroticism and a stressful situation may strongly predispose to disease and affect the severity of disease symptoms [18].

Research carried out in patients with IBD showed no significant differences on the level of such personality factors of the "Big Five" model as extroversion, agreeableness, and conscientiousness. Results regarding extroversion and conscientiousness were almost the same, but the percentage of patients with a high level of agreeableness was greater in the CD group (45\%) than in the UC group (34\%). Openness to experience, despite being statistically insignificant, was the most important differentiating factor in both groups. In the CD group up to $41 \%$ of patients achieved a high level of performance, and a low severity was found in $20 \%$ of patients. In the UC group $28 \%$ of patients showed a high score, and $24 \%$ subjects had a low level, but for most subjects moderate level features were observed. It confirms the results observed by Luban-Plozza and Poldinger, who found that patients with UC could endure new bad situations while changes at work or the environment could be contributory factors to the development of the disease [9]. A previous study [19] shows that openness, agreeableness, and conscientiousness were associated with healthy lifestyle in both sexes, while unhealthy lifestyle was positively associated with
Table IV. The intensity of the state anxiety and trait anxiety

\begin{tabular}{lcccccc} 
Disease & \multicolumn{3}{c}{ State } & \multicolumn{3}{c}{ Trait } \\
\cline { 2 - 7 } & L & M & H & L & M & H \\
\hline CD & 18 & 30 & 52 & 19 & 34 & 47 \\
\hline UC & 12 & 40 & 48 & 8 & 54 & 38
\end{tabular}

L-low level of trait (1-3 sten), M-moderate level of trait (4-6 sten), $H$ - high level of trait (7-10 sten).

neuroticism in women and extraversion in men. The demonstrated associations among conscientiousness, agreeableness, and neuroticism with health-related behaviours suggest that we might expect these domains to be related to healthier eating habits. In a study [20] comparing women with cardiovascular disease (CVD), women with cancer, and healthy women noted that neuroticism, conscientiousness, and agreeableness levels in healthy women and in those suffering from cancer was high, while in patients with CVD they were at a moderate level. In the case of openness in patients with cancer and CVD the trait level was high, whereas in healthy women it was at a moderate level. The extraversion level was high in women with CVD and moderate in the group with cancer and in the healthy group.

Anxiety and depressive symptoms are considered among the possible predictors of the development of certain diseases, including inflammatory bowel diseases [21]. Research by Kurina et al. has shown that anxiety and depression may be predictive of the development of UC in the population [22]. The association of anxiety and depression with UC was stronger if the mental state of the respondents was assessed shortly before diagnosis. Other studies have found that another important element that could lead to the emergence of IBD is inadequate defence.

Hyphantis et al. demonstrated that defence mechanisms in patients with CD turned out to be less mature than the mechanisms exhibited by patients with UC. Psychotherapy, which consists of learning to cope with chronic stress, used as an adjunct to pharmacological treatment in patients with $C D$, resulted in faster recovery and a fewer relapses compared to conventional

Table V. Assessment of the personal ability to control major emotions in patients with CD and UC

\begin{tabular}{|c|c|c|c|c|c|c|c|c|c|}
\hline \multirow[t]{2}{*}{ Disease } & \multicolumn{3}{|c|}{ Anger } & \multicolumn{3}{|c|}{ Depression } & \multicolumn{3}{|c|}{ Anxiety } \\
\hline & Low & Medium & High & Low & Medium & High & Low & Medium & High \\
\hline$C D$ & 33 & 43 & 24 & 12 & 62 & 26 & 14 & 52 & 34 \\
\hline UC & 31 & 45 & 24 & 18 & 55 & 27 & 31 & 49 & 20 \\
\hline
\end{tabular}

Low level of trait 7-14 patients; moderate level of trait 15-21 patients; high level of trait 22-28 patients. 
treatment of patients without the use of psychotherapy [23].

In our research we found that patients with IBD demonstrated considerable severity of anxiety. Almost half of the respondents in both groups received high scores in both STAI scales. The results were similar to each other and there were no statistically significant differences between them. Result indicates that the diagnosis of the level of anxiety in patients with IBD is crucial, because high levels of anxiety can exacerbate the symptoms of the disease, impede adaptation to the disease, and reduce the chances of therapy success. For comparison, in patients with coronary artery disease in which the level of anxiety is often described as a high, the percentage of people with a high level of anxiety stands at $42 \%$ [24].

The tendency to inhibit negative emotions turned out to be a differentiating factor for most respondents. Research on the emotional control scale showed that patients with IBD were characterised by a far greater emotional control than healthy people. Furthermore, patients with CD were distinguished by a higher control of negative emotions compared with those with UC. The results indicate different emotional reactions in both groups. This was confirmed in relevant literature, which presented the tendency towards the immature emotional reactions among patients with CD [23]. Similar results were observed in patients with gastroesophageal reflux disease (GERD). These patients have a great tendency to suppress negative emotions [25], and the strongest suppression of emotions is related to anxiety and depression.

The incidence of type $D$ personality in patients with IBD is higher than in the general population, where it accounts for $35 \%$ of cases [26]. The study of type D personality in people with IBD showed that more than half of respondents represented this type of personality. The percentage of people with type $D$ personality was greater among those with UC (62\%) than in patients with CD (55\%). For comparison, in patients with cardiovascular disease Type D personality is present in from 21 to $53 \%$ of patients across different diagnostic categories (e.g. acute coronary syndrome, hypertension, chronic heart failure, peripheral arterial disease, etc.).

The vast majority of patients with IBD are characterised by discomfort felt in the presence of others, concealment, and lack of social balance. These people often refrain from expressing negative emotions and behaviours associated with these emotions. The results achieved in the dimension of social inhibition have been supported by the results obtained for this group on a scale of emotional control - CECS. The data obtained are consistent with research conducted by Haller et al., who showed that people with IBD more often than not stay "closed" in their environment and have a small social support network, which is basically limited to their immediate family [27]. Negative emotionality is characterised by dysphoria, worry, and annoyance, and it is negative emotionality that has proved to be a differentiating dimension for the studied groups. High scores of NE were observed in $70 \%$ of patients in the group with UC, and in $62 \%$ of patients in the CD group. These findings coincide with the results obtained in neuroticism dimension of the Big Five model where patients with UC showed a tendency for higher severity characteristics than patients with CD [14].

A limitation of this study is the relatively small sample size, which meant that it was not possible analyse the correlation between the severity and clinical course, and the individual psychological factors.

\section{Conclusions}

It has been shown that patients with inflammatory bowel disease are characterised by personality traits considered to be a direct or predisposing factor to the occurrence of diseases. There were no statistically significant differences in terms of personality factors in the group of patients with CD and UC. We noticed, however, a higher tendency for greater neuroticism and lower openness to experience in patients with UC. It was found that the majority of patients with IBD suffer elevated or high sense of anxiety.

The study groups differed in their control of negative emotions such as anger, depression, and anxiety. Patients with CD exhibited specific inhibition of the expression of the emotions of fear, possibly reflecting the fact that patients selected for this group developed immature defence mechanisms.

The vast group of patients with inflammatory bowel disease were found to have stressful personality. Patients with UC were found to experience D personality traits more often than patients with $C D$, but the differences were not statistically significant. The duration of illness was found not to affect the factors. Almost the only element that the disease duration depended on was the feeling of anxiety as a state.

Analysis of the results suggest that in the treatment of IBD psychological therapy consisting of changing the way of disclosure of emotion may be helpful. Behavioural changes leading to modification of personality traits making up the type D personality and mastering the ability to cope with anxiety and depression could significantly improve the wellbeing of patients and hence increase satisfaction with treatment.

\section{Conflict of interest}

The authors declare no conflict of interest. 


\section{References}

1. Froch B, Zwolińska-Wcisło M, Bętkowska-Korpała B, Mach T. The dynamics of emotional reactions in patients with inflammatory bowel disease. Prz Gastroenterol 2009; 4: 141-6.

2. Kozłowska KA, Bączyk G, Krokowicz P. Quality of life in patients with ulcerative colitis treated surgically. Prz Gastroenterol 2014; 9: 220-6.

3. Bączyk G, Karoń J, Krokowicz P. The objective and subjective dimension of quality of life in patients with inflammatory bowel diseases treated on surgical wards. Prz Gastroenterol 2011; 6: 170-5.

4. Fiocchi C. Inflammatory bowel disease: etiology and pathogenesis. Gastroenterology 1998; 115: 182-205.

5. Triantafillidis JK, Merikas E, Gikas A. Psychological factors and stress in inflammatory bowel disease. Expert Rev Gastroenterol Hepatol 2013; 7: 225-38.

6. Andrzejewska J, Talarska D, Michalak M, Linke K. Quality of life in patients with Crohn's disease and ulcerative colitis. Comparative analysis. Prz Gastroenterol 2009; 4: 251-5.

7. Neurath MF. Cytokines in inflammatory bowel disease. Nat Rev Immunol 2014; 14: 329-42.

8. Birrenbach T, Bocker U. Inflammatory bowel disease and smoking: a review of epidemiology, pathophysiology, and therapeutic implications. Inflamm Bowel Dis 2004; 10: 848-59.

9. Luban-Plozza B, Pöldinger W, Kröger F. Psychosomatic disorders in general practice. $3^{\text {rd }}$ ed. Heidelberg, Springer Verlag, Berlin 1992.

10. McMahon AW, Schmitt P, Patterson JF, Rothman E. Personality differences between inflammatory bowel disease patients and their healthy siblings. Psychosom Med 1973; 35: 91-103.

11. Addolorato G, Stefanini GF, Capristo E, et al. Anxiety and depression in adult untreated celiac subjects and in patients affected by inflammatory bowel disease: a personality "trait" or a reactive illness? Hepatogastroenterology 1996; 43: 1513-7.

12. Sanchez-Munoz F, Dominguez-Lopez A, Yamamoto-Furusho JK. Role of cytokines in inflammatory bowel disease. World J Gastroenterol 2008; 14: 4280-8.

13. Lickiewicz B, Zwolińska-Wcisło M, Lickiewicz J, et al. Significance of personality features in the illness adaptation process in patients with inflammatory bowel disease. Prz Gastroenterol 2010; 5: 157-63.

14. Ogińska-Bulik N, Juczyński Z. Personality, stress and health [Polish]. Difin, Warsaw 2008.

15. Richman LS, Kubzansky L, Maselko J, et al. Positive emotion and health: going beyond the negative. Health Psychol 2005; 24: 422-9.

16. Ogińska-Bulik N, Juczyński Z. Personality traits conducive to somatic diseases - the role of type D [Polish]. Psychoonkologia 2008; 12: 7-13.

17. Mawdsley JE, Rampton DS. Psychological stress in IBD: new insights into pathogenic and therapeutic implications. Gut 2005; 54: 1481-91.

18. Bitton A, Sewitch MJ, Peppercorn MA, et al. Psychosocial determinants of relapse in ulcerative colitis: a longitudinal study. Am J Gastroenterol 2003; 98: 2203-8.

19. Brummett BH, Siegler IC, Day RS, et al. Personality as a predictor of dietary quality in spouses during midlife. Behav Med 2008; 34: 5-10.
20. Alex RA, Personality pattern of female cardiovascular patients and cancer. Patients: an analytical study. Natural and Social Sciences 2014; 2: 61-72.

21. Jaeschke R, Siwek M, Grabski B, Dudek D. Comorbidity of depression and anxiety [Polish]. Psychiatria 2010; 7: 189-97.

22. Kurina LM, Goldacre MJ, Yeates D, Gill LE. Depression and anxiety in people with inflammatory bowel disease. J Epidemiol Community Health 2001; 55: 716-20.

23. Kamrowska A, Kasprzak K, Marciniak J, et al. Anxiety and depression in ulcerative colitis and Lesniovsky-Crohn's disease [Polish]. Pol Merkur Lekarski 2010; 28: 239-41.

24. Szekely A, Balog P, Benko E, et al. Anxiety predicts mortality and morbidity after coronary artery and valve surgery: a 4-year follow-up study. Psychosom Med 2007; 69: 625-31.

25. Marcinkowska-Bachlińska M, Małecka-Panas E. The role of the type $A$ behaviour in the pathogenesis of gastroesophageal reflux disease. Prz Gastroenterol 2006; 1: 98-104.

26. Oginska-Bulik, N. Type D personality. Theory and research [Polish]. Wydawnictwo Wyższej Szkoły Humanistyczn-Ekonomicznej, Łódź 2009.

27. Haller C, Kemmler G, Judmair G, er al. Social network and social support in patients with chronic inflammatory bowel disease. Z Psychosom Med Psychother 2003; 49: 36-48.

Received: 7.01.2015

Accepted: 26.02 .2015 